\title{
Available therapeutic options following failure of a first anti-TNF agent
}

\author{
Alan J Silman
}

In the routine care of patients with rheumatoid arthritis (RA), failure of first-line treatment with anti-tumor necrosis factor (TNF) agents is not uncommon. Failure, however, is a relative term; in this therapeutic context it reflects the attainment of an insufficient level of improvement to justify continuation of therapy. With regard to health economics, failure is defined by only a modest benefit compared to the costs of the drug (and potential toxicity). Clinical failure leading to treatment cessation is based on the aspiration that an alternative therapy might prove more efficacious. In either instance, a pressing issue is the potential value in switching to a second anti-TNF agent compared to any alternative treatments. In the UK, the National Institute for Health and Clinical Excellence (NICE) recently attempted an economic appraisal of the effects of such anti-TNF switching. Its initial assessment - that the benefit was too modest relative to the cost-faced an appeal by pharmaceutical companies, together with patient and professional organizations. Consequently, NICE has agreed to reassess the issue, calling it one of 'considerable complexity' (NICE [http:// www.nice.org.uk/nicemedia/pdf/Rheumatoid ArthritisAdalimumabAppealPanelDecision.pdf] [accessed 9 January 2009]).

Indeed, there is a lack of clinical trials that compare, for example, the effect of continuing a single treatment to that of switching to a second (or even a third) anti-TNF agent, an alternative biologic (such as rituximab or abatacept), or even to standard DMARDs. There is also the assumption that the effects of switching between anti-TNF agents is independent of which agent fails, whereas it seems likely that switching between biologically similar compounds (such as monoclonal antibodies) may be less advantageous than switching to a different molecular target. Robust data relating to order effects are also elusive. Not surprisingly, it

... it is also not
unexpected
that patients
respond
variably (often
unpredictably)
to the different
agents.

\section{AJ Silman is a}

Professor of Rheumatic Disease Epidemiology and Medical Director of the Arthritis Research Campaign at the University of Manchester, Manchester, UK.

Competing interests
The author declared no
competing interests.
www.nature.com/clinicalpractice
doi:10.1038/ncprheum1028

is more difficult to show health economic benefit second time around; it is also not unexpected that patients respond variably (often unpredictably) to the different agents. Rheumatologists are anecdotally aware of the unpredictable patient response to different NSAIDs.

Where possible, however, the question of benefit should be subject to data-driven scientific scrutiny, even in the absence of sound clinical trial data. Manufacturers, who normally sponsor such trials, are reluctant to mount the large and expensive trials required to show relatively little benefit. By contrast, wellconducted observational studies can help. The British Society for Rheumatology Biologics Register studied 868 patients who responded inadequately to a first anti-TNF agent (Hyrich KL et al. [2008] Rheumatology (Oxford) 47: 10001005), comparing those who continued this treatment (persisters) with those who changed to a second anti-TNF agent (switchers) or an alternative DMARD treatment. After adjusting for confounding by indication, there was a definite improvement in Health Assessment Questionnaire score for switchers; however, this improvement was only modestly higher than that seen in persisters. In terms of economics, for equally expensive therapies, persisting and switching are similar. Indeed, restricting a prescriber's ability to change treatments could result in higher levels of persistence in 'failed' therapeutic regimens. Thus, with the likely rise in the number of licensed anti-TNF preparations, the need for a more-rational approach to prescribing increases. The hope is that large, well-conducted, observational register-based studies will identify the optimum order of treatment and subset of RA patients who would benefit from switching to another anti-TNF agent, compared to those for whom persistence, stopping or changing agent class would be more appropriate. 